\title{
Classification of Radar Echoes for Identification and Remote Reading of Chipless Millimeter-wave Sensors
}

\author{
Dominique Henry, Timothée Marchal, Julien Philippe, Patrick Pons and Hervé Aubert
}

\begin{abstract}
This paper describes the wireless and simultaneous interrogation and identification of multiple passive (zero-power) pressure sensors in an industrial environment with a reading range of at least 4 meters. The 3D beamscanning of the scene is performed from a $24 \mathrm{GHz}$ FM-CW radar and for diverse electric field polarizations. The identification is performed using a $\mathrm{k}$ nearest neighbors classification. The benefit of using the crosspolarized electric fields combined with a radar imagery technique is enlightened from the analysis of the background clutter and the simultaneous remote interrogation of several passive pressure sensors at a distance up to $17.7 \mathrm{~m}$. The measurement uncertainty on pressure obtained from the proposed long-range wireless technique is finally reported and discussed.
\end{abstract}

Index Terms - radar imaging, radar polarimetry, target classification, sensor identification, wireless sensor systems

\section{INTRODUCTION}

D ETECTION and remote reading of sensors in electromagnetic reflective and/or harsh environments is challenging, especially when sensors are chipless and batteryless (or zero-power). Paradoxically, these sensors might be very useful in such environments, in which integrated circuits may be damaged and human intervention is limited. Remote sensing using Surface Acoustic Wave tags, passive Radiofrequency identification (RFID) tags or radiofrequency (RF) resonators has been investigated in harsh conditions, such as, in extreme temperatures [1] [2] or cluttered environments [3]. The lifetime of such tags in extreme conditions depends mainly on their constitutive materials, but the reliability of the wireless link between tags and their reader may be significantly degraded by electromagnetic clutter (spurious radar echoes), multiple reflections (multipath) or destructive interferences (shadow regions). It may decrease the signalto-noise ratio and eventually make tags undetectable by the reader. One solution for enhancing the signal-to-noise ratio is to take advantage of the polarization diversity. Benefits of using cross-polarized electric fields have been investigated for chipless RFID sensor tags in [4]-[6]. However, these studies targeted short reading ranges of typically few tens of centimeters. In many industrial applications, such as Structural Health Monitoring (SHM) in inaccessible areas, larger range of wireless interrogation is often required. Cross-polarized sensor tags combined with a microwave radar imagery technique allow reaching radar-to-tag distance of 50 meters in indoor

D.Henry, T.Marchal, J.Philippe, P.Pons and H.Aubert are with LAAS-CNRS and Toulouse University, Toulouse, France.

Manuscript received July 21, 2020; environments [7]. In the paper presented at International Microwave Symposium 2020 by the authors of the present study [8], the long-range wireless multi-sensing technique in industrial environments using zero-power (passive or chipless) pressure sensors and cross-polarized electric fields was proposed. The term long-range referred to a distance of interrogation of 3 meters or more, while the term short-range referred to reading ranges of few centimeters and is usually offered by standard chipless RFID sensor tags. Moreover, the term multi-sensing referred to the simultaneous remote interrogation of multiple sensors in the scene. We investigated in [8] the radar interrogation of several passive pressure sensors surrounded by many electromagnetic reflective structures (such as metallic pipes, grids, walls, etc.) in an industrial environment. The reader was a $24 \mathrm{GHz}$ FM-CW (FrequencyModulated Continuous-Wave) radar that performed the 3D beamscanning of the scene with two orthogonal (Vertical and Horizontal) electric field polarizations in order to enhance the signal-to-noise ratio.

We report here an extension of the work reported in [8] by investigating for the first time the identification of chipless sensors from an original classification of radar echoes. Chipless sensors, such as in RFID applications, are usually identified in the frequency and/or time domains (see e.g. [9] [10] and may require the use of several transmission lines [11]. The original method proposed here does not require the use of multi-band resonators for identifying chipless sensors, but it relies only on the classification of sensor radar echoes for different field polarizations.

Classification of radar echoes is usually applied to meteorological radar imagery, synthetic aperture radar (SAR) imaging or through-the-wall radar imaging (TWRI). For SAR imaging or meteorological radar images, textural and polarimetric features can be analyzed for segmentation and classification purposes [12] [13], while on TWRI application, classifications can be applied to 3D radar images [14]. Segmentation and identification of 3D radar targets are also possible, as shown in [15]. In the application reported here, we use a classification algorithm (so-called the k-nearest neighbors [16]) to distinguish between radar echoes of millimeter-wave chipless sensors and electromagnetic clutter. The segmentation of radar echoes is performed using isolines computed with a marching square algorithm [17] in 2D azimuth/elevation planes of the radar beamscanning. The proposed classification allows to better detect and mitigate the clutter, and is also able to identify different chipless pressure sensors. To illustrate the 

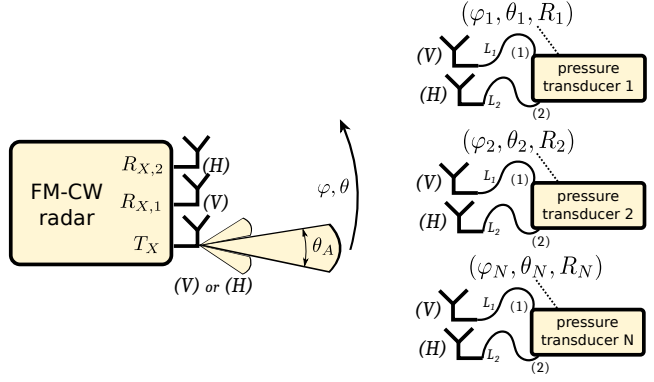

Fig. 1. Schematic of the 3D beamscanning principle of $N$ zero-power sensors with a FM-CW radar using polarization diversity. Pressure sensors are here passive pressure transducers connected to two cross-polarized antennas through delay lines.

performance of the proposed classification technique applied to sensor radar echoes for very long reading ranges $(8 \mathrm{~m}$ and more), an additional beamscanning is performed to detect, identify and read two chipless pressure sensors which are located respectively at $8.1 \mathrm{~m}$ and $17.7 \mathrm{~m}$ in front of the radar reader.

This article is organized as follows: Section II presents the main principle of the millimeter-wave radar multi-sensing technique used in this study. Some terms used throughout this paper are reminded, such as the so-called polarization configuration (Section II-A). The identification and reading algorithm is detailed in Section II-B, and Section III describes the material and methods. Scattering parameters of three pressure chipless sensors are discussed in Section III-A while the technical characteristics of the radar reader are detailed in Section III-B. Two measurement scenarii are described in Section IV-A and the method to estimate the maximal full-scale range of the pressure measurement at each sensor position is reported in Section IV-B. Descriptors are defined in Section IV-C in order to characterize the electromagnetic clutter for the two scenarii and polarization configurations. In Section IV-D, it is shown that the clutter is dramatically mitigated from the proposed analysis of segmented radar echoes. Classifications of radar echoes for different scenarii are then performed and analyzed in Section IV-E. The pressure applied on multiple chipless sensors is estimated in Section IV-F and finally the measurement uncertainty is discussed in Section IV-G.

\section{Measurement Principle}

\section{A. Multi-Sensing and Polarization Configuration}

The measurement principle is illustrated on Fig. 1. The reader unit is a FM-CW radar with carrier frequency $f_{c}$ and bandwidth $B$. The radar front-end is composed of the transmission channel $\left(T_{x}\right)$ and two reception channels $\left(R_{x, 1}\right.$ and $\left.R_{x, 2}\right)$. The $T_{x}$ antenna has narrow beamwidth in azimuth and elevation, denoted respectively by $\varphi_{A}$ and $\theta_{A}$, and its polarization is either vertical $(V)$ or horizontal $(H)$. The $R_{x, 1}$ antenna is vertically polarized, while the polarization of the $R_{x, 2}$ antenna is horizontal. The radar performs the beamscanning of the scene, in which are distributed $N$ zero power pressure (or batteryless) sensors at respective positions $\left(\varphi_{1}, \theta_{1}, R_{1}\right)$, $\left(\varphi_{2}, \theta_{2}, R_{2}\right), \ldots,\left(\varphi_{N}, \theta_{N}, R_{N}\right)$ in the elevation/azimuth coordinate system, where the origin $(0,0,0)$ is the $\mathrm{FM}-\mathrm{CW}$ radar location. The triplet $\left(\varphi_{k}, \theta_{k}, R_{k}\right)$ designates the azimuth, elevation and range from the radar of the $k^{\text {th }}$ pressure chipless sensor. As illustrated in Fig. 1, each sensor is composed of : (i) the zero-power pressure transducer, that is, a two-port passive network which converts the pressure variation applied to the sensor into the modification of its scattering parameters $S_{11}, S_{12}, S_{21}, S_{22}$ at the radar operating frequency; (ii) $\mathrm{V}$ and $H$-polarized antennas; (iii) Two delay lines of electrical lengths $L_{1}$ and $L_{2}$, which are used for connecting the $V-$ and $H$-polarized antennas to the input port (1) and output port (2) of the transducer. The analysis of the so-called backscattering antenna (or sensing) mode [18] of such passive and wireless sensors is used for remotely estimating the pressure variation applied to the sensor. This electromagnetic backscattering originates in the impedance mismatch at input and output ports of the transducer: as the mismatch level depends on the pressure applied on the transducer, pressure variation is expected to change the radar echo level of the sensor. The electric field transmitted by the radar is here either $H$ - or $V$-polarized, while the sensing mode is dual-polarized, that is, the electric field backscattered by the sensor is the combination of $H$-polarized and $V$-polarized electric fields. When the electric field transmitted by the radar is, say, $H$-polarized, the $H$-polarized component of the backscattered electric field is due to the sensing and structural modes of the $H$-polarized antenna of the sensor. Moreover, the $V$-polarized component of the field backscattered by the sensor is generated by the radiation from the $V$-polarized sensor antenna of the electric field received by the $H$-polarized sensor antenna and transmitted through the transducer and delay lines. The resulting $V$-polarized component of the backscattered electric field is next received by the $R_{x, 1}$-antenna.

TABLE I

EXPECTED DISTANCE FROM THE RADAR OF THE BACKSCATTERING SENSING MODE OF THE $k^{\text {th }}$ PRESSURE SENSOR AS A FUNCTION OF THE POLARIZATION CONFIGURATION $p$.

\begin{tabular}{|l|c|c|c|}
\hline \multicolumn{2}{|c|}{} & \multicolumn{2}{c|}{ Radar $T_{x}$ antenna polarization } \\
\cline { 3 - 4 } \multicolumn{2}{|c|}{} & $V$-pol & $H$-pol \\
\hline $\begin{array}{l}\text { Radar } R_{x} \\
\text { antennas } \\
\text { polarization }\end{array}$ & \multirow{2}{*}{-pol } & $\begin{array}{c}p=V V ; \\
R_{k}+L_{1}\end{array}$ & $\begin{array}{c}p=V H ; \\
R_{k}+\frac{L_{1}+L_{2}}{2}\end{array}$ \\
\cline { 2 - 4 } & $H$-pol & $\begin{array}{c}p=H V ; \\
R_{k}+\frac{L_{2}+\dot{L}_{1}}{2}\end{array}$ & $\begin{array}{c}p=H H ; \\
R_{k}+L_{2}\end{array}$ \\
\hline
\end{tabular}

In order to clarify the various polarization configurations studied in this paper, the so-called polarization configuration, denoted by $p$, is defined here as follows: when the electric field transmitted by the radar is $H$-polarized (resp. $V$-polarized), then :

(i) $p=H V$ (resp. $p=V V$ ) if the electric field is received by the $R_{x, 1}$-antenna ;

(ii) $p=H H$ (resp. $p=V H$ ) if the electric field is received by the $R_{x, 2}$-antenna.

Throughout this paper, $p=V V$ or $H H$ refers to the copolarization (co-pol) configuration, while $p=V H$ or $H V$ refers to the cross-polarization (cross-pol) configuration. The eventual electromagnetic coupling between the two sensors antennas is assumed to be negligible. As reported in Table I, it is expected that the distance between the radar and the 
backscattering sensing mode depends on the chosen polarization configuration.

\section{B. Identification and Remote Sensing Algorithm}

We propose an algorithm for both the detection of radar echoes associated with the chipless pressure sensors distributed in the beamscanned scene and the estimation of the pressure at the multiple sensor locations. This algorithm is composed of the following steps (see Fig. 2):

(i) Raw data is collected during the 3D mechanical radar beamscanning and for the four possible polarization configurations ( $p=V V, V H, H V$ and $H H$ ). Raw data is a $2 \mathrm{D}$ time data array. The first dimension is called the fast time, while the second dimension is called the slow time. The fast time is determined by the up-ramp duration $\left(T_{u p}=20 \mathrm{~ms}\right)$ of the radar triangular modulation frequency. $N_{S}(=1024)$ denotes the number of samples for the fast time signal. The slow time is obtained during the 3D mechanical beamscanning. The repetition time between each fast time measurement is denoted $T_{r e p}$ and is set here to $50 \mathrm{~ms}$. The direction of interrogation $\left(\varphi_{m}, \theta_{q}\right)$ is associated with each fast time signal $n$;

(ii) The Fast Fourier Transform $(F F T)$ is applied over the fast time dimension to obtain the so-called beat frequency spectrum. Positive frequencies are selected and turned into range bins of theoretical resolution $d(=7.5 \mathrm{~cm})$;

(iii) The three-dimensional data is reconstructed for the four polarization configurations. The first dimension is the interrogation range $R$. The second and third dimensions are respectively the azimuth $\theta$ and the elevation $\varphi$;

(iv) The mean patch filter of $3 \times 3 p x^{2}$ is applied on each elevation/azimuth plane. Then radar echoes are segmented for each elevation/azimuth plane $\left(\theta_{k}, \varphi_{k}\right)$. The segmentation is based on the marching squares algorithm [17] that generates contours called isolines. Isolines refer to lines along which the radar echo level does not change [19]. The echo level for which radar echoes are segmented depends on specific parameters, such as, the maximal number of local maxima inside the isoline, the initial echo level for which isolines are computed and the minimal and maximal areas inside the isoline. The following parameters are used to compute the isolines: initial echo level is $-80 \mathrm{~dB}$, the maximal number of local maxima per isolines is 1 , and the area inside an isoline ranges from $3 p x^{2}$ to $20 p x^{2}$. A more detailed description of the algorithm can be found in [20].The segmentation algorithm is not applied to the two copolarization configurations $p=V V$ and $H H$ because the lower SNR in these configurations may impact the segmentation efficiency ;

(v) Isolines are indexed with some of their features, such as their coordinates, shape or echo levels inside their boundaries. We denote by $\mathcal{C}_{i}^{p}$ the $i^{t h}$ isoline in polarization configuration $p$. As it will be justified in Section IV-D, only cross-polarization configurations $p=V H$ and $H V$ are considered in this step ;

(vi) To identify the radar echoes from the pressure sensors, only isolines $\mathcal{C}_{i}^{V H}$ and $\mathcal{C}_{j}^{H V}$ that intersect in the plane $\left(\theta_{k}, \varphi_{k}\right)$ are selected. This selection is explained for two reasons: on one hand, sensing modes for $p=V H$ and $p=H V$ are located at the same distance $R_{k}$, and on the other hand, antennas of

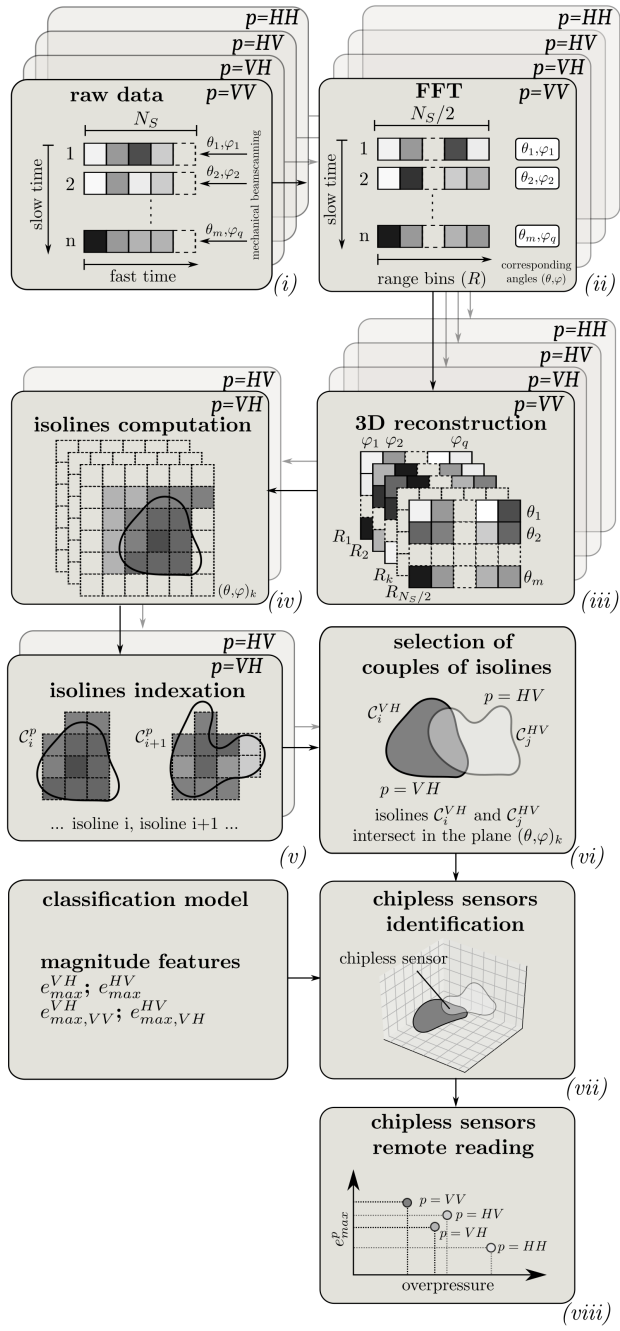

Fig. 2. The different steps of the proposed identification and remote sensing algorithm for the detection and remote reading of chipless and passive millimeter-wave sensors distributed in a cluttered environment.

chipless pressure sensors are located in close directions ;

(vii) The remaining couples of isolines are used to distinguish between radar echoes of millimeter-wave chipless sensors and electromagnetic background clutter. The standard nearest neighbors classification [21] is applied to magnitude parameters derived from isolines. The performance of the classification, which depends on the environment, will be discussed in Section IV-E ;

(viii) Once the couple of isolines associated with each chipless sensor is selected, we define the estimator $e_{\max }^{p}$ as the maximal value of echo level inside the isoline in polarization configuration $p$. This estimator varies with the pressure applied to the sensor. Cross-polarization configurations will be preferred in this study for the pressure estimation as it will be justified in Section IV-G.

\section{Material AND Methods}

\section{A. Passive Pressure Transducers}

We consider three chipless millimeter-wave pressure sensors. They are referred as sensor 1, sensor 2 and sensor 3 . These sensors are two-port wireless and passive (see [22] for 


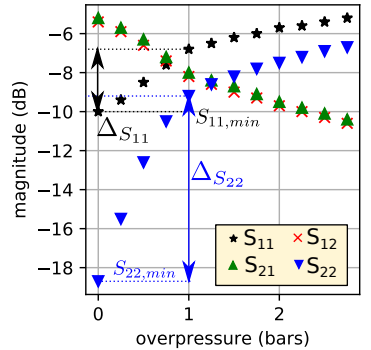

(a)

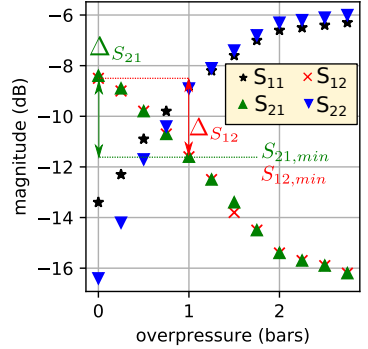

(b)

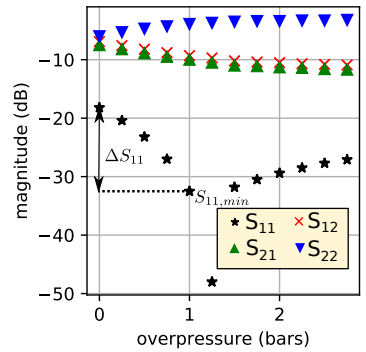

(c)

Fig. 3. Measured scattering parameters at $23.8 \mathrm{GHz}$ of (a) sensor 1 , (b) sensor 2 and (c) sensor 3 as a function of the applied overpressure. $\Delta_{S_{q}}$ and $S_{q, \min }$ (where $q=11,12,21$ and 22 ) denote respectively the full-scale range and smallest magnitude of the scattering parameter $S_{q}$ for an overpressure ranging from 0 to 1 bar.

a detailed presentation). Their respective scattering parameters at $23.8 \mathrm{GHz}$ are given in Fig. 3. For a specified interval of applied overpressures, the full-scale measurement range of radar echoes depends on the following sensor descriptors :

- the interval $\Delta_{S_{q}}$ (where $q=11,12,21$ or 22 ) in which the magnitude of scattering parameter $S_{q}$ lies ;

- the smallest magnitude $S_{q, \min }$ (where $q=11,12,21$ or 22) of the scattering parameter $S_{q}$.

If $\Delta_{S_{q}}$ is high, a large measurement range of radar echo level may be expected. Moreover, very small echo levels above the noise level can be measured for low $S_{q, \min }$. The lowest achievable $S_{q, \text { min }}$ depends on the radar sensitivity (i.e. the smallest echo level detectable by the radar) and on the electromagnetic clutter. A cluttered environment may actually cause low signal-to-noise ratio and small full-scale measurement range of radar echoes. The measurement results of descriptors $\Delta_{S_{q}}$ and $S_{q, \min }$ are reported in Table II. As it will be discussed in Section IV-F, they will significantly impact the remote sensing performances of the millimeterwave reader.

Identical cross-polarized rectangular horn antennas (gain of $20 \mathrm{dBi}$ ) are connected at input and output ports of the sensors. Since $L_{1}=L_{2}$ it can be derived from Table I that sensing modes are located at the same distance from the reader in all polarization configurations $p$. For sensor 1 and sensor 3, $L_{1}=L_{2}=0.36 \mathrm{~m}$ while for sensor $2, L_{1}=L_{2}=1.2 \mathrm{~m}$.

\section{B. The Reader}

The reader is the FM-CW radar (from IMST [23]) with carrier frequency $f_{c}=23.8 \mathrm{GHz}$ and modulation bandwidth $B=2 \mathrm{GHz}$. The theoretical depth resolution is then $d=\frac{c}{2 B}$ $(=7.5 \mathrm{~cm})$, where $c$ is the speed of light [24]. The $\operatorname{radar} T_{x}$ antenna is a circular horn with a dielectric lens, and its gain is of $28 \mathrm{dBi}$ with same beamwidth in azimuth and elevation
TABLE II

DESCRIPTORS $\Delta_{S_{q}}$ AND $S_{q, \text { min }}$ OF THE THREE CHIPLESS PRESSURE SENSORS AND FOR APPLIED OVERPRESSURE RANGING FROM 0 BAR TO 1 BAR.

\begin{tabular}{|c|c|c|c|c|c|c|}
\hline \multirow{2}{*}{$\mathrm{q}$} & \multicolumn{2}{|c|}{ sensor 1 } & \multicolumn{2}{c|}{ sensor 2 } & \multicolumn{2}{c|}{ sensor 3 } \\
\cline { 2 - 7 } & $\Delta_{S_{q}}$ & $S_{q, \text { min }}$ & $\Delta_{S_{q}}$ & $S_{q, \text { min }}$ & $\Delta_{S_{q}}$ & $S_{q, \text { min }}$ \\
\hline 11 & 3.2 & -10.0 & 4.6 & -13.4 & 14.3 & -32.5 \\
\hline 12 & 2.8 & -8.2 & 3.1 & -11.6 & 2.4 & -9.3 \\
\hline 21 & 2.8 & -8.0 & 3.2 & -11.6 & 2.5 & -10.0 \\
\hline 22 & 9.5 & -18.7 & 7.5 & -16.4 & 2.1 & -6.0 \\
\hline
\end{tabular}

all values are in $\mathrm{dB}$

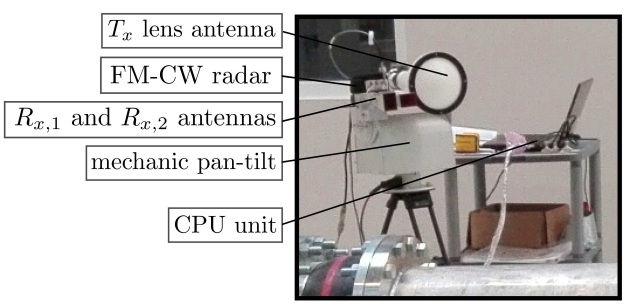

Fig. 4. FM-CW radar mounted on a pan-tilt platform. The reader performs a beamscanning in azimuth and elevation.

$\left(\varphi_{A}=\theta_{A}=6^{\circ}\right) . R_{x, 1}$ and $R_{x, 2}$ antennas are rectangular horns with a gain of $20 \mathrm{dBi}$. The output power of the radar frontend is of $10 \mathrm{dBm}(10 \mathrm{~mW})$. As depicted in Fig 4, the radar is mounted on a pan-tilt plateform performing the mechanical beamscanning. Angular resolutions both in azimuth and elevation are of $1^{\circ}$.

\section{Measurement Results And Discussion}

\section{A. Measurement Scenarii}

The remote sensing is performed at Chatou EDF research center, France (Electricité de France). This place includes an industrial hangar with a testing loop, called Everest, dedicated to the measurement of the flow and pressure inside pipes. It contains many metallic and massive objects that generate strong electromagnetic clutter. The chipless pressure sensors are interrogated in the two configurations illustrated in Fig. 5 and called scenario 1 and scenario 2. For the scenario 1 , sensor 1 and sensor 2 are placed respectively at $4.2 \mathrm{~m}$ and $4.8 \mathrm{~m}$ from the reader, as depicted in Fig. 5a and Fig. 5c. According to the electrical lengths of the delay lines (see Section III-A), sensing modes are located at ranges of $4.5 \mathrm{~m}$ and $6.0 \mathrm{~m}$ (note that in our previous contribution reported in [8], the reading range of sensor 1 is of $3.3 \mathrm{~m}$, using delay lines of electrical length $L_{1}=L_{2}=1.2 \mathrm{~m}$ ).

For the scenario 2, sensor 2 and sensor 3 are placed respectively at $8.1 \mathrm{~m}$ and $17.7 \mathrm{~m}$ from the $\mathrm{FM}-\mathrm{CW}$ radar, as depicted in Fig 5b and Fig 5d. As a result, sensing modes are located respectively at distances of $9.3 \mathrm{~m}$ and $18.8 \mathrm{~m}$ from the reader. For each scenario, the radar performs a 3D beamscanning of the scene for applied overpressures varying from 0 bar to 1 bar. 


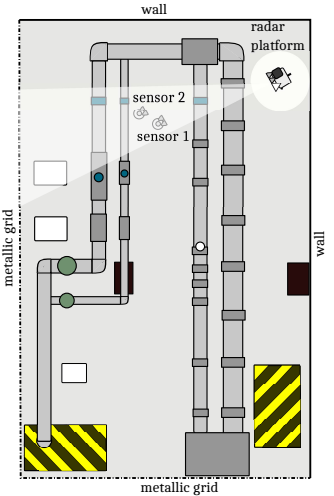

(a)

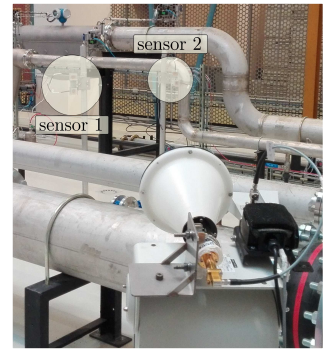

(c)

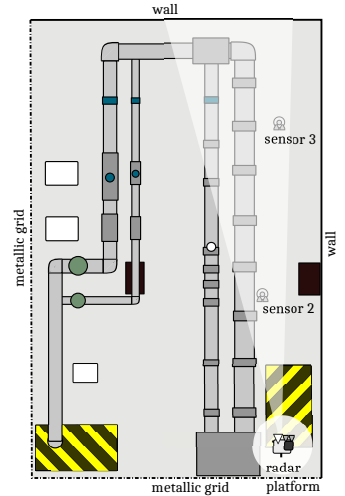

(b)

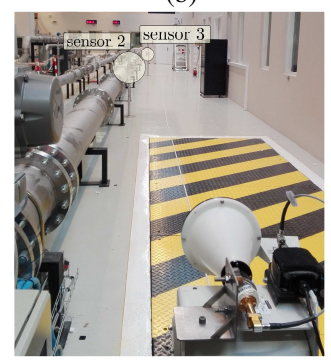

(d)
Fig. 5. Top views and pictures of (a)-(c) scenario 1 and (b)-(d) scenario 2 In scenario 1 , sensor 1 and sensor 2 are located at $4.2 \mathrm{~m}$ and $4.8 \mathrm{~m}$ from the reader. In scenario 2, sensor 2 and sensor 3 are respectively located at $8.1 \mathrm{~m}$ and $17.7 \mathrm{~m}$ from the reader.

\section{B. Characterization of the highest full-scale measurement range}

As reported in Section IV-A, the pressure chipless sensors are placed at various locations and may offer different fullscale measurement ranges. The highest full-scale measurement range $\Delta_{\max }^{p}$ (in $\mathrm{dB}$ scale) for a specified polarization configuration $p$ and fixed sensor location can be defined as follows:

$$
\Delta_{\max }^{p}=\left|e_{\max , \text { opened }}^{p}-e_{\max , \text { through }}^{p}\right|
$$

where statistical estimator $e_{\text {max, opened }}^{p}$ evaluates the highest echo level (in $\mathrm{dB}$ ) inside isolines when the two antennas of the sensor are open-circuited, and $e_{\max , \text { through }}^{p}$ estimates the highest echo levels (in $\mathrm{dB}$ ) inside isolines, when the two-port sensor is replaced by an impedance matched transmission line.

To illustrate the physical meaning of $\Delta_{\max }^{p}$, we consider the sensor 2 in the scenario 2. The corresponding sensing mode is located at a distance of $9.3 \mathrm{~m}$ from the reader. Radar echo levels at this distance are displayed in Fig. 6a for $p=V V$, $V H, H V$ and $H H$. As expected, the highest electromagnetic backscattering is obtained either from co-polarization configurations $(p=V V$ and $p=H H)$ when the sensors antennas are open-circuited, or from cross-polarization configurations ( $p=V H$ and $p=H V$ ) when the two-port sensor is replaced by an impedance-matched transmission line. The radar echoes are segmented by red isolines according to the step (iv) of the algorithm described in Section II-B. The highest echo level is given by the statistical estimator $e_{\max }^{p}$ computed from echoes inside the red isoline.

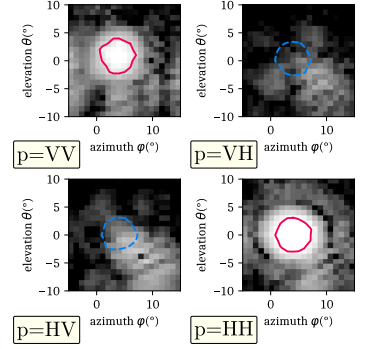

(a)

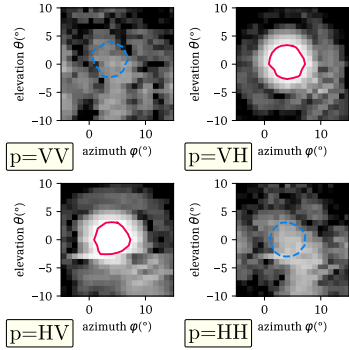

(b)
Fig. 6. Isolines generated in the azimuth/elevation planes at the range $R=9.3 \mathrm{~m}$ at the location of the sensor 2 (scenario 2) when the two-port sensor is replaced by: (a) an open-circuit at each input port of the two sensor antennas, and (b) by an impedance-matched transmission line. Dark to bright colors in the figures indicate low $(-90 \mathrm{~dB})$ to high $(-60 \mathrm{~dB})$ radar echo levels.

The lowest electromagnetic backscattering is obtained either from cross-polarization configurations $(p=V H$ and $p=H V)$ when the two-port sensor is replaced by an impedancematched transmission line, or from co-polarization configurations ( $p=V V$ and $p=H H$ ) when the sensor antennas are opencircuited. The highest echo level is then given by the statistical estimator $e_{\max }^{p}$ computed from echoes inside the blue isoline in Fig. 6. The highest full-scale measurement range $\Delta_{\max }^{p}$ is then derived from eq. 1 and radar echo levels inside the red and blue isolines.

The estimation of $\Delta_{\max }^{p}$ is performed for each sensor and each scenario. The results are reported in Table III for the four polarization configurations. For $p=V H, H V$ and open-circuited sensor antennas, we observe that the smallest value of $e_{\max }^{p}$ is lower than one obtained from $p=V V, H H$ and the transmission line between the two sensor antennas. Consequently, $\Delta_{\max }^{p}$ is higher for $p=V H$ and $H V$. This result can be explained by higher SNR offered by the crosspolarization configuration, since the scene does not strongly depolarize the electric field in our case. We note that $\Delta_{\max }^{p}$ is higher in scenario 1 than in scenario 2 because the SNR decreases with the reading range. For example, the highest full-scale measurement range $\Delta_{\max }^{H V}$ is of $20.2 \mathrm{~dB}$ for sensor 2 in scenario $l(R=4.8 \mathrm{~m})$ while $\Delta_{\max }^{V H}$ is of $14.6 \mathrm{~dB}$ in scenario $2(R=8.1 \mathrm{~m})$. Moreover, it can be also observed from Table III that, for too long reader-to-sensor distances, the detection is no more possible in co-polarization configuration, but is still achievable using cross-polarization configuration. As a matter of fact, at the position of sensor 3 (scenario $2, R=17.7 \mathrm{~m}$ ), the algorithm of Section II-B (step (iv)) does not allow the detection of radar echoes for $p=V V$ and $H H$, but it detects sensor radar echoes for $p=V H$ and $H V$. The highest full-scale measurement range is then found to be of at least $7 \mathrm{~dB}$ in this polarization configuration.

\section{Electromagnetic Clutter Analysis}

Isolines of the beamscanned scene are computed for 3D radar images in elevation and azimuth planes for the two scenarii (see step (iv) of the algorithm in Section II-B). Computed isolines associated with the clutter of scenario 1 are displayed in Fig. 7 in black color for $p=V V, V H, H V$, and $H H$ in $(\theta, \varphi, R)$ coordinate system. It can be observed 
TABLE III

HIGHEST FULL-SCALE MEASUREMENT RANGE $\Delta_{\text {max }}^{p}$ FOR ALL SENSOR POSITIONS AND POLARIZATION CONFIGURATIONS.

\begin{tabular}{|c|c|c|c|c|c|c|}
\hline \multirow[b]{2}{*}{$\mathrm{p}$} & \multicolumn{3}{|c|}{ sensor 1} & \multicolumn{3}{|c|}{ sensor 2} \\
\hline & $o$ & - & $\Delta_{\max }^{p}$ & $o$ & - & $\Delta_{\max }^{p}$ \\
\hline$V V$ & -52.1 & -61.6 & 9.6 & -54.0 & -64.5 & 10.5 \\
\hline$V H$ & -73.3 & -53.1 & 20.2 & -72.4 & -53.5 & 18.9 \\
\hline$H V$ & -76.2 & -52.6 & 23.6 & -73.6 & -53.5 & 20.2 \\
\hline$H H$ & -52.4 & -58.1 & 5.6 & -54.2 & -70.9 & 16.7 \\
\hline \multicolumn{7}{|c|}{ scenario 1} \\
\hline & \multicolumn{3}{|c|}{ sensor 2} & \multicolumn{3}{|c|}{ sensor 3} \\
\hline $\mathrm{p}$ & $O$ & - & $\Delta_{\max }^{p}$ & $O$ & - & $\Delta_{\max }^{p}$ \\
\hline$\overline{V V}$ & -58.7 & -70.2 & 11.5 & ND & ND & 0 \\
\hline$V H$ & -70.8 & -56.2 & 14.6 & -64.3 & -57.1 & 7.2 \\
\hline$H V$ & -66.2 & -55.1 & 11.1 & -67.7 & -58.9 & 8.8 \\
\hline$H H$ & -55.0 & -65.5 & 10.5 & ND & ND & 0 \\
\hline
\end{tabular}

all values are in $\mathrm{dB}$ scale

$o$ : open-circuited two-por

- : matched-impedance transmission line

ND: radar echo not detected

that the number of isolines differs significantly between copol and cross-pol configurations: the number of isolines is actually of 722 for $p=V V, 445$ for $p=V H, 462$ for $p=H V$, and 716 for $p=H H$. This is due to the reduction of the clutter in the cross-pol radar images compared with one obtained from co-pol radar images. In red and blue colors are displayed in Fig. 7 the isolines of sensing modes of sensor 1 and sensor 2. During the beamscanning, the applied overpressures were of 0,7 bar for sensor 1 , and 1,1 bars for sensor 2 . The computed isolines allow estimating the location of these sensors with angular resolutions of $1^{\circ}$ in azimuth and elevation, and theoretical range resolution of $7.5 \mathrm{~cm}$. Inside the volume of the 3D scene beamscanned by the radar in a given polarization configuration, the sensor-to-clutter radar echoes ratio $\Lambda_{p}$ is computed as follows :

$$
\Lambda_{p}=\frac{N_{s, p}}{N_{s, p}+N_{c, p}}
$$

where $p$ denotes the polarization configuration, $N_{s, p}$ is the number of isolines generated by the sensors and $N_{c, p}$ is the number of isolines generated by the clutter. In absence of clutter in the beamscanned volume, $\Lambda_{p}$ is equal to 1 and all isolines in this volume are generated only by the sensing mode. Consequently, the applied overpressure can be derived from the analysis of the isolines, as it will be shown below. However, when the clutter is very high, $\Lambda_{p}$ is close to 0 and the isolines are generated by the clutter only. As a consequence, the sensing modes are no more detectable and the overpressure cannot be remotely estimated. Therefore, $\Lambda_{p}$ characterizes the ability to identify the sensor radar echo in a given beamscanned scene and polarization configuration. If the comparison of the number of clutters is needed between two beamscanned scenes, $N_{c, p}$ must be normalized by the number of pixels contained in the scene (or in the scanned volume). The scanned volume in elevation/azimuth/range coordinates is defined by :

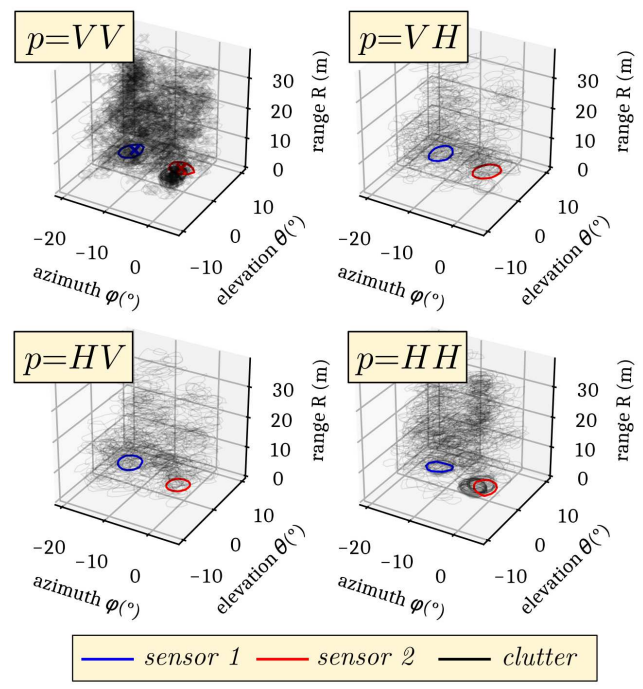

Fig. 7. Isolines (in elevation/azimuth/range coordinates) derived from the radar beamscanning of scenario 1 and computed for different polarization configurations $(p=V V, V H, H V$, and $H H)$. In black color are displayed isolines of the clutter, while blue and red colors indicate isolines of the sensing modes for respectively sensor 1 (for an applied overpressure of 0,7 bar) and sensor 2 (for an applied overpressure of 1,1 bars).

$$
V_{\text {scan }}=\frac{1}{3}\left(R_{1}^{3}-R_{0}^{3}\right) \times\left(\varphi_{1}-\varphi_{0}\right) \times\left(\sin \left(\theta_{1}\right)-\sin \left(\theta_{0}\right)\right)
$$

where $R_{1}$ and $R_{0}$ denote the highest and smallest reading ranges, $\varphi_{1}$ and $\varphi_{0}$ the highest and smallest azimuth angles, $\theta_{1}$ and $\theta_{0}$ highest and smallest elevation angles. We derive that $V_{\text {scan }}=2826 \mathrm{~m}^{3}$ for scenario 1 and $V_{\text {scan }}=2261 \mathrm{~m}^{3}$ for scenario 2. The number of detected radar echoes from the clutter per unit volume is given by :

$$
N_{V c, p}=\frac{N_{c, p}}{V_{s c a n}}
$$

From the computed isolines, the following sensor-to-clutter radar echoes ratios and the number of isolines generated by the clutter per unit volume are derived for the two scenarii and reported in Table IV. In scenario 1, the cross-pol ratios $\Lambda_{V H}$ and $\Lambda_{H V}$ are nearly twice times larger than the copol ratios $\Lambda_{V V}$ and $\Lambda_{H H}$. Therefore, compared with the copol configurations, the cross-pol configurations allow detecting and identifying more easily the backscattering sensing modes in presence of strong electromagnetic clutter. Inversely in scenario $2, \Lambda_{V H}$ and $\Lambda_{H V}$ are lower than $\Lambda_{V V}$ and $\Lambda_{H H}$. This result can be explained from observation of the pictures of scenario 2 on Fig. 5b and Fig. 5d. Sensor 2 and sensor 3 are actually located close to a long metallic pipe with circular riveted joints. These circular shapes depolarize the electric field. This interpretation is confirmed by the highest value of $N_{V c, p}$ obtained for $p=V H$ or $H V$ in scenario 2 compared with one provided by scenario 1 .

\section{Clutter Filtering using Intersection of Isolines}

To facilitate the classification of radar echoes and filter part of the clutter, only isolines of radar echoes in crosspolarization configurations $p=V H$ and $H V$ that intersect are selected, as indicated in step (vi). This selection is possible 
TABLE IV

SENSOR-TO-CLUTTER RADAR ECHOES RATIO $\Lambda_{p}$ AND NUMBER OF ISOLINES GENERATED BY THE CLUTTER PER UNIT VOLUME $N_{V, c, p}$ FOR THE TWO SCENARII IN ALL POLARIZATION CONFIGURATIONS.

\begin{tabular}{|c|c|c|c|c|}
\hline \multirow{2}{*}{$\mathrm{p}$} & \multicolumn{2}{|c|}{ scenario 1} & \multicolumn{2}{c|}{ scenario 2} \\
\cline { 2 - 5 } & $\Lambda_{p}$ & $N_{V, c, p}{ }^{a}$ & $\Lambda_{p}$ & $N_{V, c, p}{ }^{a}$ \\
\hline$V V$ & $2.8 \mathrm{E}-3$ & $2.5 \mathrm{E}-1$ & $11.6 \mathrm{E}-3$ & $0.7 \mathrm{E}-1$ \\
\hline$V H$ & $4.5 \mathrm{E}-3$ & $1.5 \mathrm{E}-1$ & $4.4 \mathrm{E}-3$ & $2.0 \mathrm{E}-1$ \\
\hline$H V$ & $4.3 \mathrm{E}-3$ & $1.6 \mathrm{E}-1$ & $4.5 \mathrm{E}-3$ & $1.9 \mathrm{E}-1$ \\
\hline$H H$ & $2.8 \mathrm{E}-3$ & $2.5 \mathrm{E}-1$ & $13.2 \mathrm{E}-3$ & $0.6 \mathrm{E}-1$ \\
\hline
\end{tabular}
$N_{s, p}=2$
${ }^{u}$ unit in radar echoes per $m^{3}$

because of the close positions of the two cross-polarized antennas for each chipless sensor. Consequently, radar echoes of the sensing modes for $p=V H$ and $H V$ are located in close elevation and azimuth angles at a given reading range. This initial filtering is illustrated in Fig. 8 for the scenario 2 at the location of sensor $3(R=18.1 \mathrm{~m})$. In Fig. 8a and Fig. 8b are represented isolines for respectively $p=V H$ (blue isolines) and $p=H V$ (green isolines). By transposing these isolines in the same azimuth/elevation plane, we select the isolines for $p=V H$ and $p=H V$ that intersect. The remaining couples of isolines are shown in Fig. 8c. We note that the third green isoline for $p=H V$ has been removed/filtered because no other isoline intersects this isoline for $p=V H$. We observe that the first remaining couple of isolines allows segmenting the sensing mode of sensor 3. The other one is used to segment the unfiltered clutter.

To evaluate the efficiency of this filtering, the sensor-toclutter radar echoes ratio and number of isolines generated by the clutter per unit volume, defined respectively by eqs (2) and (4), are re-computed after the step (vi) of the algorithm. These parameters are denoted by $\Lambda_{(V H) \cap(H V)}$ and $N_{V, c,(V H) \cap(H V)}$. For scenario $1, \Lambda_{(V H) \cap(H V)}=9.1 \mathrm{E}-3$ and $N_{V, c,(V H) \cap(H V)}=7.6 \mathrm{E}-2$ radar echo per $m^{3}$. For scenario 2, $\Lambda_{(V H) \cap(H V)}=8.0 \mathrm{E}-3$ and $N_{V, c,(V H) \cap(H V)}=1.0 \mathrm{E}-1$ radar echo per $m^{3}$. By comparing these values with the ones for $p=V H$ and $p=H V$ in Table IV, we observe that around $50 \%$ of the clutter has been removed by filtering in both scenarii.

\section{E. Classification of Radar Echoes}

In order to identify radar echoes of the chipless sensors, a classification of radar echoes is now proposed using the measurement results obtained from scenario 1, scenario 2 and for various overpressures applied to the sensors. The classifier used here for the identification is a standard nearest neighbors classifier [21]. In contrast to previously reported identification techniques (see e.g. [9]-[11]), the proposed method does not used resonators or other passive structures for remotley identifying chipless sensors. The method proceeds only to the classification of sensor radar echoes for the four polarization configurations.

Four statistical estimators, or features, are considered here for the classification:

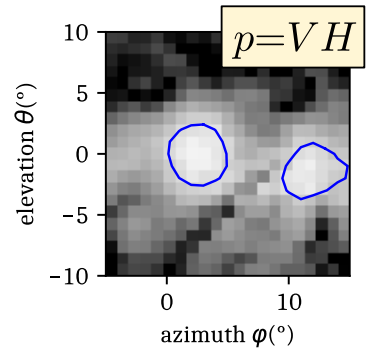

(a)

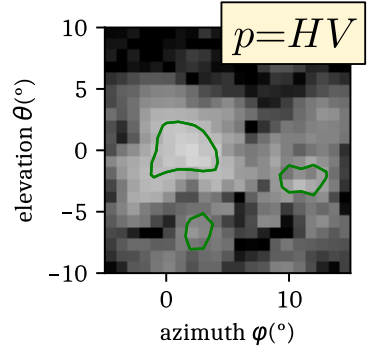

(b)

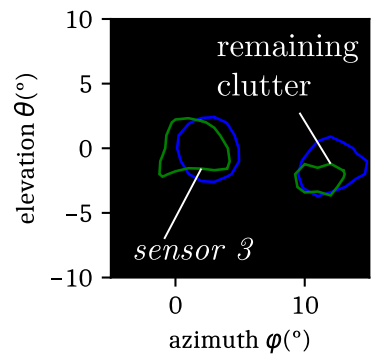

(c)

Fig. 8. Isolines generated in the azimuth/elevation planes at the range $R=18.1 \mathrm{~m}$ at the location of the sensor 3 (scenario 2) for (a) $p=V H$ and (b) $p=H V$. Dark to bright colors indicate low $(-90 \mathrm{~dB})$ to high $(-60 \mathrm{~dB})$ echo levels. (c) Only couples of isolines that intersect are selected.

- $e_{\max }^{V H}$, which estimates the highest echo level inside the isoline $\mathcal{C}_{i}^{V H}$ from the selected couple of intersected isolines $\left(\mathcal{C}_{i}^{V H}, \mathcal{C}_{j}^{H V}\right)$;

- $e_{\text {max }}^{H V}$, which estimates the highest echo level inside the isoline $\mathcal{C}_{j}^{H V}$ from the selected couple of intersected isolines $\left(\mathcal{C}_{i}^{V H}, \mathcal{C}_{j}^{H V}\right)$;

- $e_{\max , V V}^{V H}$, which estimates the highest echo level inside the isoline $\mathcal{C}_{i}^{V H}$ from the selected couple of intersected isolines $\left(\mathcal{C}_{i}^{V H}, \mathcal{C}_{j}^{H V}\right)$ transposed in the same elevation/azimuth plane for $p=V V$;

- $e_{\max , H H}^{H V}$, which estimates the highest echo level inside the isoline $\mathcal{C}_{j}^{H V}$ from the selected couple of intersected isolines $\left(\mathcal{C}_{i}^{V H}, \mathcal{C}_{j}^{H V}\right)$ transposed in the same elevation/azimuth plane for $p=H H$;

Information about the couple of intersected isolines are referred in Section IV-D. To perform the classification, the classifier takes into consideration the 3 nearest neighbors from the couple of intersected isolines to be tested. The following classifications are here trained and tested :

- The first classification is trained with couples of isolines from scenario 1 for various applied overpressures between 0 bar and 1 bar. Classes 0,1 and 2 designate respectively couples of isolines of the clutter, sensor 1 and sensor 2;

- The second classification is trained with couples of isolines from scenario 2 for various applied overpressures between 0 bar and 1 bar. Classes 0,2 and 3 designate respectively couples of isolines of the clutter, sensor 2 and sensor 3;

- The third classification is trained with couples of isolines from both scenario 1 and scenario 2 for various applied overpressures between 0 bar and 1 bar. Classes $0,1,2$ and 3 designate respectively couples of isolines of the 
clutter, sensor 1, sensor 2 and sensor 3.

For each case, $50 \%$ of the beamscanning data are used to build the classes, the remaining $50 \%$ are used to test the classes. Since the four features $\left(e_{\max }^{V H}, e_{\max }^{H V}, e_{\max , V V}^{V H}\right.$ and $\left.e_{\max , H H}^{H V}\right)$ are expected to depend on the applied overpressure, the training data includes low (0 bar) and high (1 bar) applied overpressures. Consequently, extreme values of the estimators are included in the training. In the left side of Fig. 9 is plotted $e_{\max , V V}^{V H}$ as a function of $e_{\max , H H}^{H V}$ and in the right of the figure is plotted $e_{\max }^{V H}$ as a function of $e_{\max }^{H V}$. Data of scenario 1 are indicated by circles and data of scenario 2 are plotted using crosses. Features of the clutter, sensor 1, sensor 2 and sensor 3 are displayed respectively in black, green, blue and red colors. The features of the three sensors are distinguishable. The observed variations of the feature values for a given sensor is due to the variation of the overpressure. The difference of the feature values between each sensor originates from the different scattering parameters reported in Fig. 3. We note that $e_{\max , V V}^{V H}$ of sensor 2 is shifted between scenario 1 (blue circles) and scenario 2 (blue crosses). This shift is due to lower SNR provided by scenario 2 . The most challenging issue is to distinguish between radar echoes of the sensors and the clutter. As observed, the number of data from the clutter is large and the features exhibit a very large variation ranging from $-80 \mathrm{~dB}$ to $-40 \mathrm{~dB}$.

Confusion matrices of the classifications are reported in Table V. For the first classification (scenario 1), 5 beamscannings are used for the training, and 5 other for the test. Despite the large number of clutter data, the classification is easily performed. For the second classification (scenario 2), 8 beamscannings are used for the training, and 8 other for the test. All radar echoes of sensor 2 are identified. For sensor 3, the rate of identification success reaches $75 \%$, with 6 successes, 1 failure (the radar echo is viewed as a clutter), and 1 non-detection during the step (iv) of the algorithm (this non-detection does not appear in the confusion matrix). The identification failures originate in the low SNR at the position of sensor 3 located at the distance of $17.7 \mathrm{~m}$. During the test of the second classification, sensor 3 is successfully identified. We observe that few spurious radar echoes due to the clutter is viewed as radar echoes of the sensors $(0.04 \%$ for the training, $0.23 \%$ for the test). For the third classification using data for both scenario 1 and scenario 2, conclusions are similar to ones obtained from the previous classifications. One radar echo of sensor 2 is not identified because there is a shift of $e_{\max , V V}^{V H}$ between scenario 1 and scenario 2. Moreover, the number of radar echoes from the clutter which are wrongly detected as sensor radar echoes is very small $(0.09 \%$ for the training and $0.17 \%$ for the test) and as expected, there is no false identification between the three sensors because their features can be distinguishable.

\section{F. Overpressure Estimation for Various Polarization Configu- rations}

The overpressure applied to the sensors can be derived from the isolines which have been identified as sensor radar echoes. In Fig. 10, the statistical estimator $e_{\max }^{p}$ is plotted as a function of the applied overpressure for all polarization configurations

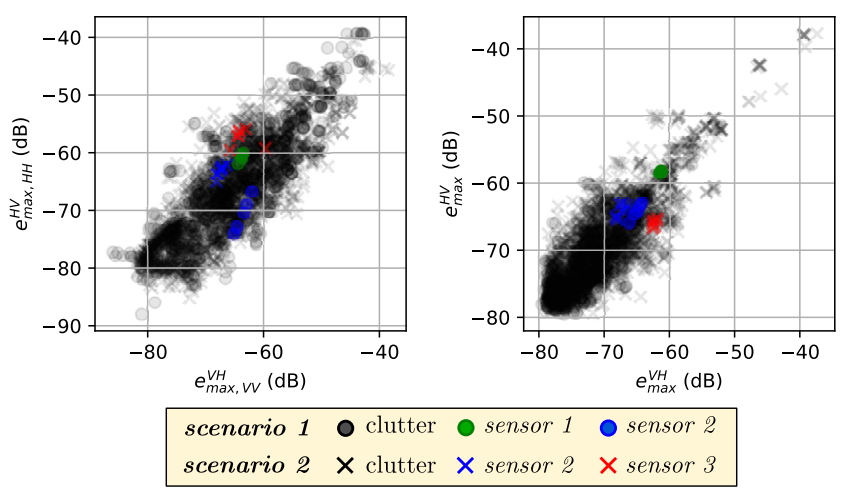

(a)

(b)

Fig. 9. (a) $e_{\max , H H}^{H V}$ as a function of $e_{\max , V V}^{V H}$ and (b) $e_{\max }^{H V}$ as a function of $e_{\max }^{V H}$ of the data used to train the classification. Data of scenario 1 are represented with circles. Data of scenario 2 are represented with crosses. Features of the clutter, sensor 1 , sensor 2 and sensor 3 are represented respectively in black, green, blue and red colors.

TABLE V

CONFUSION MATRICES OF TESTED AND TRAINED DATA FOR THE DIFFERENT SCENARII

\begin{tabular}{|c|c|c|c|c|c|c|c|}
\hline & \multicolumn{4}{|c|}{$\begin{array}{c}\text { trained } \\
\text { predicted }\end{array}$} & \multicolumn{2}{|c|}{$\begin{array}{c}\text { tested } \\
\text { predicted }\end{array}$} \\
\hline & & 0 & 1 & 2 & 0 & 1 & 2 \\
\hline \multirow{3}{*}{ actual } & 0 & 873 & 0 & 0 & 887 & 0 & 0 \\
\hline & 1 & 0 & 5 & 0 & 0 & 5 & 0 \\
\hline & 2 & 0 & 0 & 5 & 0 & 0 & 5 \\
\hline
\end{tabular}

total: 10 beamscannings

scenario 1

\begin{tabular}{|c|c|c|c|c|c|c|c|}
\hline & \multicolumn{4}{|c|}{$\begin{array}{c}\text { trained } \\
\text { predicted }\end{array}$} & \multicolumn{2}{|c|}{$\begin{array}{c}\text { tested } \\
\text { predicted }\end{array}$} \\
\hline & & 0 & 2 & 3 & 0 & 2 & 3 \\
\hline \multirow{3}{*}{ actual } & $\overline{\mathbf{0}}$ & 2328 & 1 & 0 & 2565 & 4 & 2 \\
\hline & 2 & 0 & 8 & 0 & 0 & 8 & 0 \\
\hline & 3 & 1 & 0 & 6 & 0 & 0 & 8 \\
\hline
\end{tabular}

total: 16 beamscannings

scenario 2

\begin{tabular}{|c|c|c|c|c|c|c|c|c|c|}
\hline & \multicolumn{5}{|c|}{$\begin{array}{c}\text { trained } \\
\text { predicted }\end{array}$} & \multicolumn{3}{|c|}{$\begin{array}{c}\text { tested } \\
\text { predicted }\end{array}$} \\
\hline & & $\mathbf{0}$ & 1 & 2 & 3 & $\mathbf{0}$ & 1 & 2 & 3 \\
\hline \multirow{4}{*}{ actual } & $\mathbf{0}$ & 3190 & 1 & 2 & 0 & 3507 & 0 & 4 & 2 \\
\hline & 1 & \begin{tabular}{l|}
0 \\
\end{tabular} & 5 & 0 & 0 & 0 & 5 & 0 & 0 \\
\hline & 2 & 1 & 0 & 12 & 0 & 1 & 0 & 12 & 0 \\
\hline & 3 & 1 & 0 & 0 & 6 & 0 & 0 & 0 & 8 \\
\hline
\end{tabular}

total: 26 beamscannings

scenarii $1 \& 2$

and for scenario 1 and scenario 2. Horizontal lines with the letter $\mathcal{N}$ indicate the expected lowest echo level for each sensor and scenario calculated in Section IV-B (see Table III). When a radar echo is not detected for a given applied overpressure, the default value of $e_{\max }^{p}$ is set to the expected lowest echo level $\mathcal{N}$.

To evaluate the performance of the overpressure estimation for a specified polarization configuration $p$, we define the fullscale range $\Delta_{p}$ of the echo level for each sensor and the 
sensitivity $\alpha_{p}=\frac{\Delta_{p}}{d \mathcal{P}}$ within the overpressure interval $d \mathcal{P}$. The highest $\alpha_{p}$, the more sensitive the chipless pressure sensor. To compare the efficiency of each polarization configuration, we define also the ratio $\frac{\Delta_{p}}{\Delta_{S_{q}}}$, where $\Delta_{S_{q}}$ is the full-scale range of the scattering parameters within the overpressure interval $d \mathcal{P}$. These parameters are reported in Table VI and the full-scale range $\Delta_{p}$ is indicated in Fig. 10 when it is relevant.

Sensor 1 in scenario 1 offers a high sensitivity $\alpha_{V V}=0.75$ within the overpressure range $d \mathcal{P}=0.9$ bar. We note variations of $e_{\max }^{p}$ for sensor 1 (scenario 1 ) below the expected lowest echo level $\mathcal{N}$ for $p=V V$ and $H H$. Two reasons may explain this observation: (1) the two open-circuited sensor antennas used in Section IV-B do not provide here the smallest achievable echo level, and/or (2) the scene has changed between the calibration and the overpressure measurement. We observe also that the ratio $\frac{\Delta_{H H}}{\Delta_{S_{22}}}=0.30$ is lower than one obtained from other polarization configurations. Despite the large full-scale range $\Delta_{S_{22}}$ of $7.5 \mathrm{~dB}$, the lowest magnitude of scattering parameter $S_{22, \min }=-18.7 \mathrm{~dB}$ is too low to ensure a high full-scale measurement range of echo level (see Table II). Moreover, sensor 2 in scenario 1 offers equivalent or higher ratio $\frac{\Delta_{p}}{\Delta_{S_{q}}}$ in cross-polarization configuration than in copolarization configuration. This result is explained by higher SNR and larger $\Delta_{S_{q, \min }}$. However, the pressure estimation is not possible for $p=H H$ because most of the radar echoes are not detected.

When the reading range increases (sensor 2 in scenario $2)$, the ratio $\frac{\Delta_{p}}{\Delta_{S}}$ decreases in all polarization configurations. The ratio is still higher for $p=V H$ and $p=H V$ compared with $p=V V$ and $p=H H$ despite lower value of $\Lambda_{p}$ (see Table IV). The full-scale measurement range of radar echo level is less impacted in cross-polarization configuration than in co-polarization configuration when the range of interrogation increases. Inversely to scenario 1 , the pressure estimation for $p=H H$ is possible. It means that the undetected radar echoes in scenario 1 are due to the clutter.

Moreover, no pressure estimation is possible when using sensor 3 in scenario 2 because parameters $\Delta_{S_{q, \min }}$ are too low for the reading range of $17.7 \mathrm{~m}$ (see Table II). Nevertheless, highest full-scale ranges $\Delta_{\max }^{V H}$ of $7.2 \mathrm{~dB}$ and $\Delta_{\max }^{V H}$ of $8.8 \mathrm{~dB}$ are achievable (see Table III).

\section{G. Measurement Uncertainty of the Radar Echo Level for all Polarization Configurations}

To evaluate the measurement uncertainty on the radar echo level, the beamscanning of scenario $l$ is successively performed 50 times. The mean value and standard deviation of the echo level, as well as isolines, are derived in linear scale for all (sensors and clutter) isolines. The mean value and standard deviation of $e_{\max , p}$ are computed for each isoline and displayed in Fig. 11a in dB scale. Each circle represents a radar echo measured 50 times. Red and blue colors designate respectively the cross and co-polarization configurations. It can be observed that mean $\left(e_{\max , p}\right)$ and $\operatorname{std}\left(e_{\max , p}\right)$ are strongly correlated, and mean $\left(e_{\max , p}\right)$ increases with $\operatorname{std}\left(e_{\max , p}\right)$.

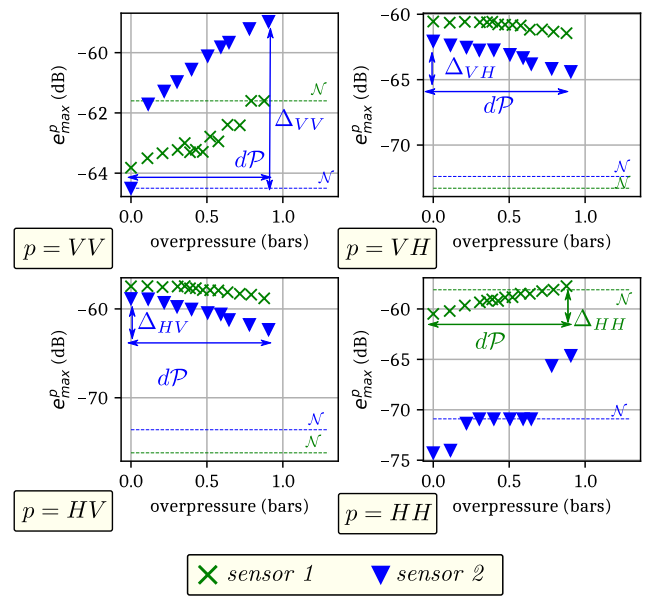

(a)
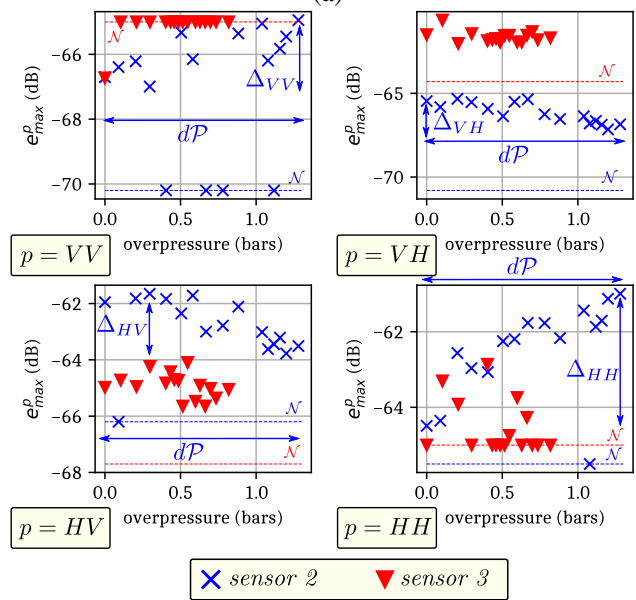

(b)

Fig. 10. Statistical estimator $e_{\max }^{p}$ as a function of the applied overpressure for $p=V V, V H, H V$ and $H H$ : (a) scenario 1 with sensor 1 (green crosses) and sensor 2 (blue triangles) and (b) scenario 2 with sensor 2 (blue crosses) and sensor 3 (red triangles). Horizontal lines labeled with the letter $\mathcal{N}$ represent the expected lowest echo level for each sensor and scenario.

Let define the measurement uncertainty $\epsilon_{p}$ on the measured echo level as follows:

$$
\epsilon_{p}=\frac{\operatorname{std}\left(e_{\max , p}\right)}{\operatorname{mean}\left(e_{\max , p}\right)}
$$

The measurement uncertainty $\epsilon_{p}$ (in $\%$ ) is displayed on Fig $11 \mathrm{~b}$ as a function of mean $\left(e_{\max , p}\right) . \epsilon_{p}$ decreases with mean $\left(e_{\max , p}\right)$ and as expected, the overpressure estimation derived from isolines of low radar echo level is less accurate than estimations obtained from isolines of higher echo level. However, compared with co-polarization configurations, the impact on measurement uncertainty is strongly reduced for cross-polarization configurations. The mean value of $\epsilon_{p}$ is plotted with black dashed lines: $\epsilon_{p}=13.7 \%$ for $p=V V$ and $p=H H$ and $\epsilon_{p}=7.3 \%$ for $p=V H$ and $p=H V$. As an example for sensor 2 , it can be observed from Fig 10a that $e_{\max , p}$ varies between $-60 \mathrm{~dB}$ and $-70 \mathrm{~dB}$. From Fig. 11b, we derived that the mean value of $\epsilon_{p}$ is $7.7 \%$ for $p=V V$ and $H H$ in this range of echo level. The measurement uncertainty on the radar echo level decreases in cross-polarization configurations: 
TABLE VI

PERFORMANCE OF THE CHIPLESS PRESSURE SENSORS FOR ALL POLARIZATION CONFIGURATIONS AND SCENARII

\begin{tabular}{|c|c|c|c|c|c|c|c|c|}
\hline \multirow{2}{*}{$\mathrm{p} / S_{q}$} & \multicolumn{4}{|c|}{ sensor 1 } & \multicolumn{5}{c|}{ sensor 2} \\
\cline { 2 - 9 } & $\Delta_{p}$ & $\Delta_{S_{q}}$ & $\alpha_{p}$ & $\frac{\Delta_{p}}{\Delta_{S_{q}}}$ & $\Delta_{p}$ & $\Delta_{S_{q}}$ & $\alpha_{p}$ & $\frac{\Delta_{p}}{\Delta_{S_{q}}}$ \\
\hline$V V / S_{11}$ & 2.2 & 2.9 & 2.5 & 0.75 & 2.8 & 4.2 & 3.1 & 0.66 \\
\hline$V H / S_{12}$ & 0.9 & 2.5 & 1.0 & 0.36 & 2.3 & 2.7 & 2.5 & 0.85 \\
\hline$H V / S_{21}$ & 1.3 & 2.5 & 1.5 & 0.52 & 3.4 & 2.8 & 3.8 & 1.21 \\
\hline$H H / S_{22}$ & 2.7 & 9.0 & 3.1 & 0.30 & $\mathrm{NR}^{a}$ & 6.9 & $\mathrm{NR}^{a}$ & $\mathrm{NR}^{a}$ \\
\hline \multicolumn{4}{|c|}{$d \mathcal{P}=0.9 \mathrm{bar}$} & \multicolumn{3}{c}{$=0.9 \mathrm{bar}$}
\end{tabular}

scenario 1

\begin{tabular}{|c|c|c|c|c|c|c|c|c|}
\hline \multirow{2}{*}{$\mathrm{p} / S_{q}$} & \multicolumn{4}{|c|}{ sensor 2} & \multicolumn{5}{|c|}{ sensor 3 } \\
\cline { 2 - 9 } & $\Delta_{p}$ & $\Delta_{S_{q}}$ & $\alpha_{p}$ & $\frac{\Delta_{p}}{\Delta_{S_{q}}}$ & $\Delta_{p}$ & $\Delta_{S_{q}}$ & $\alpha_{p}$ & $\frac{\Delta_{p}}{\Delta_{S_{q}}}$ \\
\hline$V V / S_{11}$ & 2.0 & 4.6 & 1.6 & 0.43 & $\mathrm{NR}^{a}$ & 8.0 & $\mathrm{NR}^{a}$ & $\mathrm{NR}^{a}$ \\
\hline$V H / S_{12}$ & 1.8 & 3.1 & 1.4 & 0.58 & $\mathrm{NR}^{a}$ & 1.8 & $\mathrm{NR}^{a}$ & $\mathrm{NR}^{a}$ \\
\hline$H V / S_{21}$ & 2.1 & 3.2 & 2.1 & 0.65 & $\mathrm{NR}^{a}$ & 1.9 & $\mathrm{NR}^{a}$ & $\mathrm{NR}^{a}$ \\
\hline$H H / S_{22}$ & 3.5 & 7.5 & 2.7 & 0.46 & $\mathrm{NR}^{a}$ & 1.6 & $\mathrm{NR}^{a}$ & $\mathrm{NR}^{a}$ \\
\hline \multicolumn{4}{|c|}{$d \mathcal{P}=1.3$ bars } & \multicolumn{3}{c}{$=0.8 \mathrm{bar}$}
\end{tabular}

scenario 2

$\Delta_{p}$ in dB; $\Delta_{S_{q}}$ in dB; $\alpha_{p}$ in dB per bar; $\frac{\Delta_{p}}{\Delta_{S_{q}}}$ in $\mathrm{dB}$ per dB

${ }^{a} \mathrm{NR}$ : not relevant

the mean value of $\epsilon_{p}$ is $5.8 \%$ for $p=V H$ and $H V$ for $-70 \mathrm{~dB} \leq e_{\max , p}<-60 \mathrm{~dB}$.

In Fig. 11c is displayed the sensor-to-reader distance $R$ as a function of $\epsilon_{p}$. In co-polarization configuration, we observe that the measurement uncertainty increases with $R$. This effect is not apparent in cross-polarization configuration. As an illustrative example, for the sensor-to-reader distances of $15 \mathrm{~m}$ and $20 \mathrm{~m}$, the mean value of $\epsilon_{p}$ is of $12.45 \%$ for $p=V V$ and $H H$ while $\epsilon_{p}$ is lower (7.2\%) for $p=V H$ and $H V$.

\section{CONClusion}

We have demonstrated in this paper that cross-polarized electric fields combined with a 3D radar imagery technique can be advantageously used to perform both the identification and the long-range ( $>3$ meters) reading of passive and wireless sensors in cluttered environments. Using a classification algorithm, we have also demonstrated the feasibility to efficiently distinguish between sensor radar echoes and the clutter. Different chipless and passive pressure sensors have been successfully identified in a cluttered environment and remotely read by using polarimetric features. For long reading ranges of interrogation ( $>8$ meters), it is shown that crosspolarization configuration should definitively be preferred to the copolarization configuration.

The proposed 3D analysis of the electromagnetic clutter from isolines can be advantageously applied to avoid shadow regions in the scene when distributing the chipless millimeterwave sensors. In future work, sensors allowing the simultaneous measurement of various physical quantities, such as pressure, temperature and humidity, will be investigated from the proposed remote sensing technique. Moreover, limits of the classification method will be studied to determine the maximal number of identifiable chipless sensors.

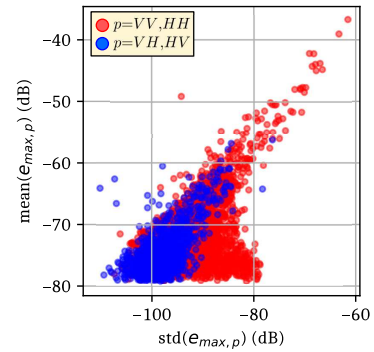

(a)

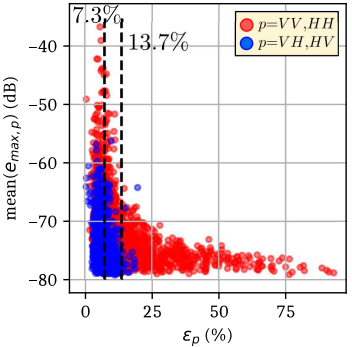

(b)

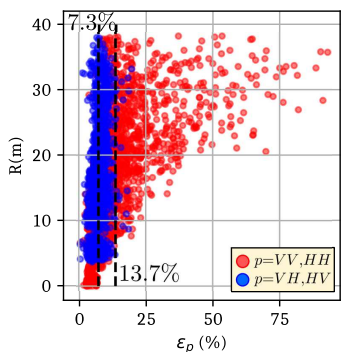

(c)

Fig. 11. Mean value mean $\left(e_{\max , p}\right)$ of the statistical estimator $e_{\max , p}$ as a function of (a) standard deviation $\operatorname{std}\left(e_{\max , p}\right)$ and (b) the measurement uncertainty $\epsilon_{p}$ defined in Eq. (5). (c) Sensor-to-reader distance in function of $\epsilon_{p}$. Isolines are computed from 50 3D radar beamscannings of scenario 1 . Red and blue circles indicate isolines obtained from respectively co-polarization and cross-polarization configurations.

\section{ACKNOWLEDGMENT}

The authors wish to thank Occitanie Regional Council (CARANUC Project) and Electricité de France (EDF) company for financial support. This work was partly supported by LAAS-CNRS micro and nanotechnologies platform members of the French RENATECH network.

\section{REFERENCES}

[1] M. Pereira da Cunha, R. J. Lad, T. B. Pollard, D. McCann, E. McCarthy, P. Prata, and R. Kelley, "Wireless harsh environment SAW array system for power plant application," in 2014 IEEE International Ultrasonics Symposium, Sep. 2014, pp. 381-384.

[2] H. Cheng, X. Ren, S. Ebadi, Y. Chen, L. An, and X. Gong, "Wireless passive temperature sensors using integrated cylindrical resonator/antenna for harsh-environment applications," IEEE Sensors Journal, vol. 15 , no. 3, pp. 1453-1462, March 2015.

[3] A. J. Mercer, R. K. James, G. Bennett, P. Patel, C. Johnston, and J. Cai, "RFID testing and evaluation for an RF-harsh environment," in 2011 IEEE International Conference on RFID-Technologies and Applications, Sep. 2011, pp. 95-102.

[4] Y. Feng, L. Xie, Q. Chen, and L. Zheng, "Low-cost printed chipless RFID humidity sensor tag for intelligent packaging," IEEE Sensors Journal, vol. 15, no. 6, pp. 3201-3208, June 2015.

[5] A. Vena, E. Perret, and S. Tedjini, "A compact chipless RFID tag using polarization diversity for encoding and sensing," in 2012 IEEE International Conference on RFID (RFID), April 2012, pp. 191-197.

[6] D. Girbau, A. Ramos, A. Lazaro, S. Rima, and R. Villarino, "Passive wireless temperature sensor based on time-coded UWB chipless RFID tags," IEEE Transactions on Microwave Theory and Techniques, vol. 60, no. 11, pp. 3623-3632, Nov 2012.

[7] D. Henry, J. G. D. Hester, H. Aubert, P. Pons, and M. M. Tentzeris, "Long-range wireless interrogation of passive humidity sensors using van-atta cross-polarization effect and different beam scanning techniques," IEEE Transactions on Microwave Theory and Techniques, vol. 65 , no. 12 , pp. 5345-5354, Dec 2017.

[8] D. Henry, T. Marchal, J. Philippe, P. Pons, and H. . Aubert, "Long-range zero-power multi-sensing in industrial environment using polarization diversity and 3d radar imagery," in International Microwave Symposium, to be published, Los Angeles, CA, 2020. 
[9] A. Vena, E. Perret, and S. Tedjini, "Chipless RFID tag using hybrid coding technique," IEEE Transactions on Microwave Theory and Techniques, vol. 59, no. 12, pp. 3356-3364, 2011.

[10] F. Chebila, M. M. Jatlaoui, P. Pons, and H. Aubert, "Reconfigurable multi-band scatterers for micro-sensors identification," in 2009 IEEE Antennas and Propagation Society International Symposium, 2009, pp. $1-4$.

[11] M. M. Jatlaoui, F. Chebila, S. Bouaziz, P. Pons, and H. Aubert, "Original identification technique of passive EM sensors using loaded transmission delay lines," in The 40th European Microwave Conference, 2010, pp. $1106-1109$.

[12] C. D'Elia, S. Ruscino, M. Abbate, B. Aiazzi, S. Baronti, and L. Alparone, "Sar image classification through information-theoretic textural features, mrf segmentation, and object-oriented learning vector quantization," IEEE Journal of Selected Topics in Applied Earth Observations and Remote Sensing, vol. 7, no. 4, pp. 1116-1126, 2014.

[13] L. Sadouki and B. Haddad, "Classification of radar echoes with a textural-fuzzy approach: an application for the removal of ground clutter observed in Sétif (Algeria) and Bordeaux France sites," International Journal of Remote Sensing, vol. 34, no. 21, pp. 7447-7463, 2013.

[14] B. G. Mobasseri and Z. Rosenbaum, "3d classification of throughthe-wall radar images using statistical object models," in 2008 IEEE Southwest Symposium on Image Analysis and Interpretation, 2008, pp. $149-152$.

[15] C. Debes, J. Hahn, A. M. Zoubir, and M. G. Amin, "Target discrimination and classification in through-the-wall radar imaging," IEEE Transactions on Signal Processing, vol. 59, no. 10, pp. 4664-4676, 2011.

[16] J. L. Bentley, "Multidimensional binary search trees used for associative searching," Commun. ACM, vol. 18, no. 9, p. 509-517, sep 1975. [Online]. Available: https://doi.org/10.1145/361002.361007

[17] W. E. Lorensen and H. E. Cline, "Marching cubes: A high resolution 3d surface construction algorithm," ACM Computer Graphics, vol. 21, no. 4, p. 163-169, 1987.

[18] R. F. Harrington, "Theory of loaded scatterers," Proceedings of the Institution of Electrical Engineers, vol. 111, no. 4, pp. 617-623, 1964.

[19] D. Henry and H. Aubert, "Isolines in 3d radar images for remote sensing applications," in 2019 16th European Radar Conference (EuRAD), 2019, pp. 69-72.

[20] D. Henry, H. Aubert, and T. Véronèse, "Proximal radar sensors for precision viticulture," IEEE Transactions on Geoscience and Remote Sensing, vol. 57, no. 7, pp. 4624-4635, 2019.

[21] "Nearest neighbors - scikit-learn 0.23 .1 documentation," https:// scikit-learn.org/stable/modules/neighbors.html, accessed: 2020-06-25.

[22] J. Philippe, M. V. De Paolis, D. Henry, A. Rumeau, A. Coustou, P. Pons, and H. Aubert, "In-situ wireless pressure measurement using zero-power packaged microwave sensors," Sensors, vol. 19, no. 6, 2019.

[23] "IMST Radar small and flexible radar modules by IMST," http://www. radar-sensor.com/, accessed: 2020-06-24.

[24] S. O. Piper, "Receiver frequency resolution for range resolution in homodyne FMCW radar," in Conference Proceedings National Telesystems Conference 1993, June 1993, pp. 169-173.

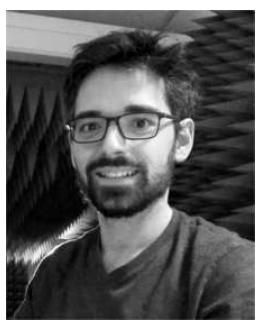

Dominique Henry Dominique Henry received the M.S. degree in electronical engineering and the $\mathrm{Ph} . \mathrm{D}$. degree from the Institut National Polytechnique de Toulouse, Toulouse, France, in 2012 and 2018, respectively. From 2013 to 2014, he was a Research Engineer with LAAS-CNRS, Toulouse. In 2014, he served in collaboration with LAAS-CNRS and Ovalie-Innovation, Auch, France. His research interests include the use of microwave radars for agricultural applications, remote sensing of passive sensors, radar interrogation techniques, and development of smart antennas in constraint environments and microwave imaging. He works now with LAAS-CNRS as a post-doctoral researcher.

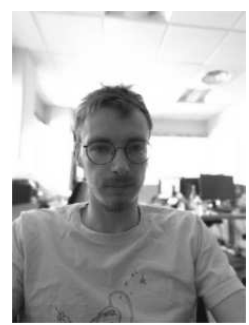

Timothée Marchal Timothée Marchal was born in France, in 1994. He graduated from the Polytechnic National Institute of Toulouse (INPT) in 2018, where he specialized in microwave engineering. He is now studying in his second year of $\mathrm{PhD}$ degree whose main subject is radar sensing for wireless sensor interrogation.

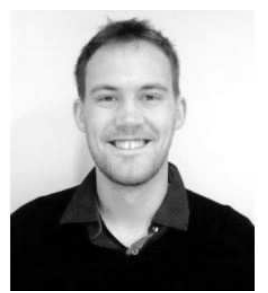

Julien Philippe Dr Julien Philippe received the M.S. degree in microelectronics and nanotechnology from the Grenoble INP PHELMA, France, in 2011. He received the Ph.D. degree in nanoelectronics and nanotechnology from the Grenoble Alpes University, France, in 2014. His work was focused on the conception, characterization and development of co-integrated NEMS (Nano Electro Mechanical Systems) - CMOS cells for gas sensing and mass spectrometry application. From February 2016 he is contracted as a Post-Doctoral Researcher in the research laboratory of analysis and architecture of systems (LAAS-CNRS) of Toulouse, France. He currently works on the fabrication and characterization of passive RF sensors based on millimeter wave transducers for wireless monitoring of physical properties.

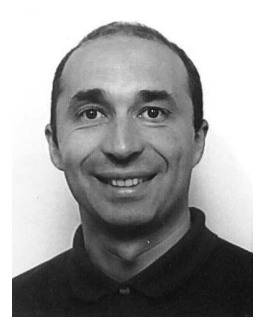

Patrick Pons Dr Patrick Pons was born in Toulouse (France) in May 1963. He received, from the Toulouse (France) University, a master in Physics in 1985 with a specialization in micro-electronics in 1986 and the $\mathrm{PhD}$ degree in electronics in 1990. Since 1991 he is a researcher in the CNRS-LAAS (National Scientific Research Center - Laboratory of Analysis and Analysis of Systems) in Toulouse. His research interests are focused in micro-technologies and micro-sensors. Dr. Patrick PONS is author or co-author of more than 95 papers in referred International Journals and more than 267 communications in International Conferences. He holds 3 international patents in the area of micro-sensors and has performed 3 technological transfers with industrial partners. He was involved in 79 funded projects including 13 as coordinator (22 International, $31 \mathrm{Na}-$ tional, 12 Regional, 14 Industrial). Dr. Patrick PONS has been the advisor or co-advisor of 30 Ph.D students. Dr. Patrick PONS was member of the steering Committee of "Micro Mechanics Europe" International Conference from 2009 to 2013. He organized "Eurosensors" International Conference in 1994 and was chairman of "Micro Mechanics Europe" International Conference in 2009. He was member of several Program Committees for International Conferences (MME, IEEE sensors, Sensornet, Eurosensors) and is reviewer for International Journals (Sensors \& Actuators, Journal of Micromechanics and Microengineering, Microelectronic Engineering, Microsystem Technology). $\mathrm{He}$ is also expert for the French Research Agency (ANR) since 2011. 


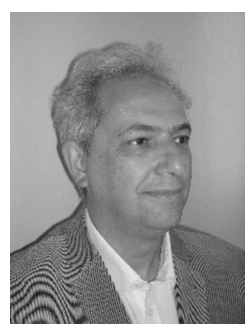

Hervé Aubert Hervé Aubert was born in Toulouse, France, in July 1966. He received the Eng. Dipl. in July 1989 and the Ph.D. degree (with highhonors) in January 1993, both in Electrical Engineering and both from the Institut National Polytechnique (INPT), Toulouse, France. From April 1997 to March 1998 he was a Visiting Associate Professor at the School of Engineering and Applied Science, University of Pennsylvania, Philadelphia, USA. Since February 2001 Hervé Aubert is Professor at INPT. He has joined the Laboratory for the Analysis and Architecture of Systems (LAAS), National Center for Scientific Research (CNRS), Toulouse, in February 2006. Since January 2015 he is the Head of the Micro- and Nano-systems for Wireless Communications Research Group at LAAS-CNRS. Dr. Aubert has performed research works on integral-equation and variational methods applied to electromagnetic wave propagation and scattering. Currently his research activities involve the electromagnetic modelling of complex (multi-scale) structures, and the wireless electromagnetic sensors. He has authored or co-authored 98 papers in indexed journals and more than 200 communications in International Symposium Proceedings, one book (in French, 2003) and 2 book chapters [Fractals: Theory and Applications in Engineering (Springer, 1999) and New Trends and Concepts in Microwave Theory and Techniques (Research Signpost, 2003)]. He holds 9 international patents in the area of antennas and wireless sensors. Dr. Aubert serves as a Subject Editor in-Chief (since 2020) and Subject Editor (since 2016) of Electronics Letters, a member of the editorial board of International Journal of Antennas and Propagation (since 2014), and he was a member of the editorial board of International Journal of Microwave Science and Technology from 2010 to 2017. He serves as a Meta-reviewer for European Conference on Antennas and Propagation since 2017. He was the member of the Technical Programme Committee of many international conferences, including the European Microwave Week from 2013 to 2019,

IEEE International Symposium on Robotic and Sensors Environments in 2019, International Symposium on Medical Information and Communication Technology in 2018 and 2019, IEEE Conference on RFID Technologies and Applications in 2014, and the Symposium on Antenna Technology and applied ElectroMagnetics in 2012. Dr. Aubert received the Best Reviewer Award of Information \& Communications Technology (ICT) Express in 2019, and the European Microwave Association Award in Recognition of Distinguished Service as General Chairman of the European Microwave Week 2015. He was the Secretary of IEEE Antennas and Propagation French Chapter from 2009 to 2013, the Vice-Chairman of this Chapter from 2004 to 2009 and Secretary from 2001 to 2004 . 